\title{
Haematology of Down syndrome
}

\author{
David Webb, Irene Roberts, Paresh Vyas
}

Arch Dis Child Fetal Neonatal Ed 2007;92:F503-F507. doi: 10.1136/adc.2006.104638

Down syndrome is a common congenital disorder affecting $\sim 1 / 1000$ live births. Newborns and children with Down syndrome may present with many haematological problems. In addition, benign abnormalities of the blood count and blood film, which may manifest at any age, population-based and cancer-based registries and clinical trials suggest there is a $\sim 12$-fold increased risk of acute lymphoblastic leukaemia in the age group of 5-30 years that rises to $\sim 40$-fold in children younger than 5 years, and that there is a $\sim 150$-fold increased risk of acute myeloid leukaemia in children younger than 5 years. There is also a virtually unique predisposition to a transient neonatal leukaemia, known as transient abnormal myelopoiesis. Deaths from leukaemia, in part, account for the excess mortality associated with Down syndrome. This article reviews the clinical presentation and the progress made in the management of these disorders over the past decade. It also briefly considers the recent exciting scientific advances that have potential to transform management of leukaemia in children with Down syndrome and also have implications for management of childhood leukaemia more generally.

See end of article for authors' affiliations

Correspondence to:

Paresh Vyas, Department of Haematology and MRC

Molecular Haematology

Unit, John Radcliffe Hospital and Weatherall Institute of Molecular Medicine,

Oxford OX3 9DU, England; paresh.vyas@imm.ox.ac.uk

Accepted 19 March 2007
M any abnormalities related to blood count have been reported in small retrospective studies in the neonatal period in Down syndrome (box 1). ${ }^{12}$ Most of these do not require referral to a specialist paediatric haematologist. Many neonates with Down syndrome, like those with trisomy 13 and trisomy 18, have haematological features typical of those seen in chromosomally normal infants with intrauterine growth restriction (IUGR): mild, transient neutropenia and thrombocytopenia together with increased numbers of circulating nucleated red cells with or without polycythaemia. ${ }^{4}$ In the setting of IUGR these changes are secondary to chronic intrauterine hypoxia. ${ }^{4}$ Blast cells (ie, early less differentiated blood cells) are not increased in this situation and confusion with transient abnormal myelopoiesis (TAM) is not a problem (see below for more about TAM). Neonates with Down syndrome may have normal blood counts, with the only abnormality being subtle dysplastic features of the white cells, platelets and/or red cells ${ }^{12}$ (fig lA). These features are often noted as incidental findings. They are probably not clinically relevant, although the ongoing large prospective studies to define these changes more precisely may provide insight into their prognostic relevance. The clinically most important abnormality is the disorder known as transient abnormal myelopoiesis or TAM.
TRANSIENT ABNORMAL MYELOPOIESIS

TAM, also called transient myeloproliferative disorder and transient leukaemia, is a haematological disorder virtually confined to Down syndrome and presents during fetal life or in the neonatal period. An identical disorder with the same natural history can also be found in neonates without Down syndrome but who have an acquired trisomy 21 confined to the haematopoietic cells. ${ }^{5}$ A similar, but distinct, disorder can be seen in some neonates with Noonan syndrome. ${ }^{6}$ However, true TAM is strictly associated with trisomy 21 . The importance of TAM is its potential to transform into an acute leukaemia, known as myeloid leukaemia of Down syndrome (ML-DS), which is estimated to occur in $\sim 20-30 \%$ of babies with TAM, although the exact frequency is not known. ${ }^{78}$ TAM can therefore be considered as a leukaemic or "pre"-leukaemic syndrome (reviewed by Zipursky ${ }^{7}$ ). Clinically, TAM is conventionally defined by a combination of its haematological and clinical features (see box 2 , and discussed in detail below). Using these criteria, about $10 \%$ of all newborns with Down syndrome have TAM. However, molecular genetic studies have recently shown that neonates with TAM have mutations in the key megakaryocyte transcription factor GATAl, ${ }^{9-13}$ which offers the opportunity to more accurately identify the true incidence of TAM in Down syndrome, since some infants with TAM do not have symptoms and may not previously have been diagnosed as having the condition. All cases of TAM should be referred to a paediatric haematologist because the neonate may need specialised intervention and also to ensure the appropriate tests are done to clinch the diagnosis (see below). Another reason for referral is that follow-up is required as there is risk of the infant developing ML-DS later.

\section{Clinical findings}

TAM has a variable clinical presentation. ${ }^{7}$ Although it may occur during fetal life, it mostly presents after birth. During gestation it usually presents with hydrops fetalis and anaemia. There is often tissue infiltration with blast cells (hepatosplenomegaly and myocardial infiltration). The outlook for TAM presenting in fetal life seems to be poor compared with cases presenting after birth $^{14}$ although spontaneous resolution of TAM in utero has been described.

After birth, a diagnosis of TAM can mostly be made simply on the basis of circulating blast cells with or without a mild leucocytosis. ${ }^{8}$ The

Abbreviations: ALL-DS, acute lymphoblastic leukaemia of Down syndrome; MDS, myelodysplastic phase; ML-DS, myeloid leukaemia of Down syndrome; TAM, transient abnormal myelopoiesis 


\section{Box 1 Haematological abnormalities in Down} syndrome

Neonates

- Transient abnormal myelopoiesis

- Non-specific changes associated with intrauterine growth restriction and trisomies: neutropenia, thrombocytopenia, erythroblastosis and polycythaemia

- Subtle myelodysplastic features: abnormal myeloid cell granulation, giant platelets

Infants and children

- Myeloid leukaemia of Down syndrome

- Myelodysplasia

- Acute myeloid leukaemia

Other: many non-malignant haematological abnormalities have been reported; their clinical importance remains to be defined. They include polycythaemia due to cardiac disease, reduced numbers of $B$ lymphocytes ${ }^{3}$ and thrombocytopenia

remainder present with any combination of the following: hydrops, a greatly elevated white blood cell count with circulating blasts, bruising, skin infiltrates, exudative effusions (pleural, pericardial and ascites), respiratory distress and hepatomegaly. Liver dysfunction with jaundice may be severe and in rare cases leads to fatal hepatic failure secondary to fibrosis. However, most cases of TAM resolve spontaneously within 3 months after birth.

\section{Laboratory findings}

The haemoglobin level and neutrophil count are usually normal in TAM, but the platelet count is often abnormal-both thrombocytopenia and thrombocytosis are reported. The blood film may show nucleated red cells, giant platelets and megakaryocyte fragments. The characteristic and invariable feature on the blood film is the presence of deeply basophilic immature blast cells (fig 1B). These are usually indistinguishable from the blast cells that are seen frequently in preterm babies. However, they are present in greater numbers than in healthy neonates and, unlike blast cells in neonates without Down syndrome, TAM blast cells persist for several weeks or months. Therefore, although the diagnosis of TAM may not be clear in the first few days of life, particularly in a preterm baby, there is usually no diagnostic difficulty once the baby is a few weeks old.

One helpful investigation in doubtful cases is flow cytometry to characterise the immunophenotype of the blast cell population. Blast cells in TAM have been shown to express early myeloid (CD34, CD33), megakaryocyte (CD41, CD61) and erythroid (CD235a, glycophorin A) antigens and may express CD7 (a T-cell antigen). Cytochemical stains can also be used to characterise the blast cells (they stain positive for acid phosphatases and non-specific esterase and negative for myeloperoxidase, Sudan black, chloroacetate esterase and period acid-Schiff) but this test is rarely used in clinical practice now. Diagnostic difficulty is common in infants with phenotypically normal mosaic Down syndrome in which the only clue to the diagnosis is a blood film picture typical of TAM. Therefore any infant with blood film abnormalities suggestive of TAM should have cytogenetic analysis to look for trisomy 21. The bone marrow findings mirror those in blood, and add little
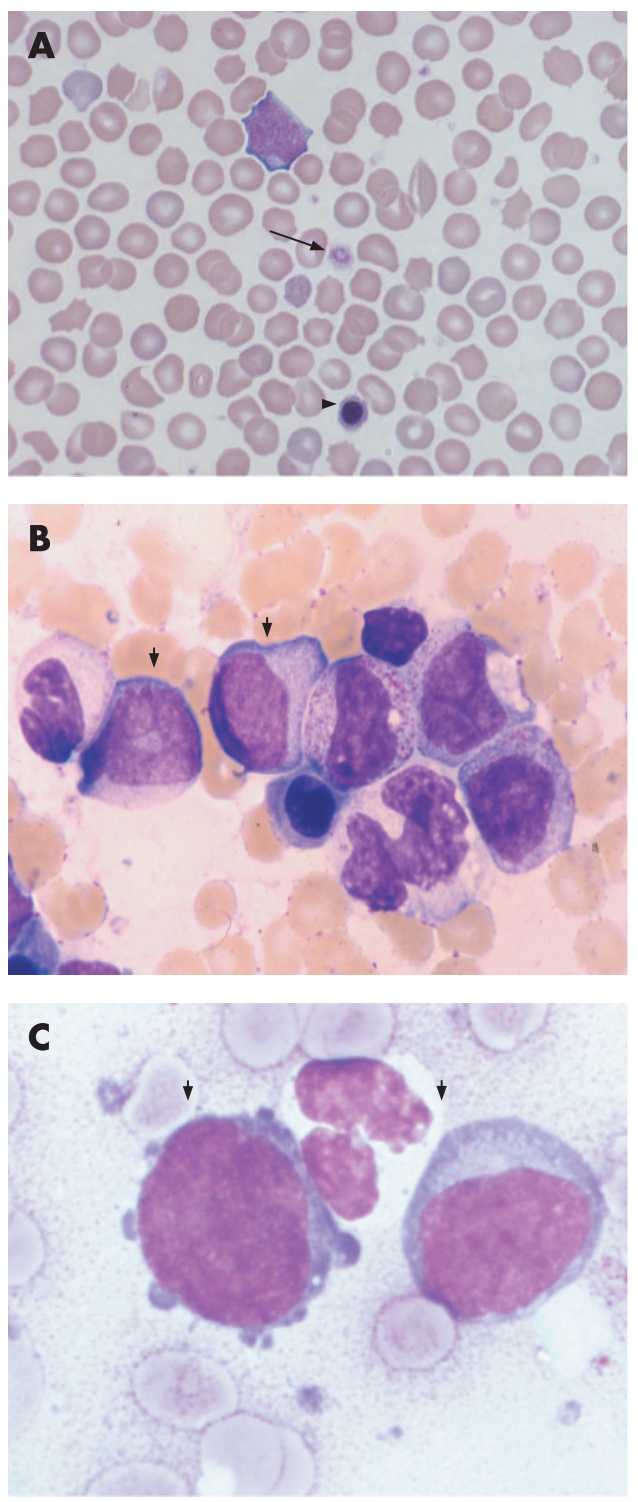

Figure 1 (A) Wright-Giemsa stained blood film of a newborn with Down syndrome showing macrocytic red cells and thrombocytopenia with a large platelet (arrow); there is also a nucleated red cell (arrowhead) $(\times 40$ magnification). (B) Bone marrow sample from a newborn with transient neonatal leukaemia stained with May-Grünwald-Giemsa showing blast cells (small arrows), more mature myelocytes, band form neutrophils and a nucleated red cell ( $\times 40$ magnification). (C) Morphologically similar blasts (arrowheads) from a May-Grünwald-Giemsa stained bone marrow sample from a child with myeloid leukaemia of Down syndrome $(\times 100$ magnification).

where the diagnosis is indicated by clinical findings and examination of the blood.

\section{Treatment}

Most children with TAM only need observation as the clinical and laboratory abnormalities spontaneously resolve within 3 months after birth. However, symptomatic babies, especially those with high blast counts or liver dysfunction may benefit from low-dose cytosine arabinoside ( $10-20 \mathrm{mg} / \mathrm{m}^{2} /$ day for up to 7 days). As TAM blasts are highly sensitive to cytarabine, there is generally a rapid response. Severe liver disease, with fibrosis due to the production of megakaryocyte-derived growth factor from blast cells, has a poor prognosis and may not respond to treatment. Despite resolution in most cases of TAM, up to $20 \%$ of infants who present to haematology centres still die of disease. $^{8}$ 
Box 2 Clinical and laboratory features of transient neonatal leukaemia in Down syndrome

Cardiopulmonary

- Pericardial effusion

- Ascites

- Pulmonary oedema

Liver

- Hepatosplenomegaly

- Hepatic fibrosis

- Liver failure

- Obstructive jaundice

Haematological

- Leucocytosis (but white blood cell count may be normal)

- Persistent peripheral blood blast cells

- Abnormal platelet count: often reduced or raised (but may be normal)

- Haemoglobin: may be reduced, raised or normal

Other

- Skin rash (vesicopapular)

Although 10\% of neonates with Down syndrome are said to develop TAM and 10\% of fetuses with Down syndrome die in utero, possibly due to TAM, the true incidence of TAM is unknown. Neonates with more subtle presentations of TAM probably escape notice as blood counts and smears are not routinely performed on all neonates with Down syndrome. To date, there have been retrospective studies ${ }^{12}$ and one small prospective study from a specialised paediatric unit (and not representative of the Down syndrome population as a whole $)^{15}$ documenting blood cell abnormalities at birth. Thus, there is a need for a prospective study on a large population of unselected newborns with Down syndrome documenting full blood counts and blood films. Such studies are ongoing in the UK, USA, Ireland and Europe. The UK study, coordinated by Oxford and York, is currently recruiting centres. Further information about this study is available from the corresponding authors. Until data are available from these studies we recommend taking full blood counts and blood films in all neonates with Down syndrome.

\section{ML-DS: A UNIQUE CLINICAL ENTITY LINKED TO TAM Clinical features}

The spectrum of acute myeloid leukaemia in Down syndrome is markedly distinct from the acute myeloid leukaemia that develops in children without Down syndrome, and the disease is now recognised as a specific entity (ML-DS) in the proposed World Health Organization (WHO) classification. ${ }^{16}$ ML-DS usually presents at between 1 and 4 years of age with a median age of presentation of 1.8 years. ${ }^{17}$ Although not all cases of ML-DS have a clinically evident preceding TAM phase, retrospective studies suggest that $\sim 20-30 \%$ of infants with TAM develop ML-DS either by overt progression or more commonly, after an apparent remission. ${ }^{18}$ This suggests that $\sim 2-3 \%$ of children with Down syndrome develop ML-DS (given that retrospective studies suggest that $\sim 10 \%$ of neonates with Down syndrome develop TAM, see above for references). However, there is a discrepancy between the incidence figures from retrospective studies and the rate of incidence of $\sim 0.75 \%$ of ML-DS in children with Down syndrome from populationbased studies. ${ }^{19}$ This discrepancy underlines the need for prospective studies following a large cohort of neonates and children with Down syndrome.

An antecedent myelodysplastic (MDS) phase is present in $70 \%$ of infants, in which the infant becomes progressively anaemic and thrombocytopenic with dysplastic changes in erythroid cells and megakaryocytes. The marrow often becomes increasing difficult to aspirate due to hypercellularity and myelofibrosis. Delay in treatment in a well child does not compromise later outcome of chemotherapy.

\section{Laboratory findings}

The blood typically shows reduced numbers of normal cells, with dysplastic changes in all myeloid lineages, and circulating blasts. The bone marrow aspirate and trephine show dysplasia, increased blasts, abnormal megakaryocytes and variable myelofibrosis. The trephine is especially important, as aspiration of marrow is often difficult or impossible. The morphological, immunophenotypic and cytochemical profile of the blasts is similar to that of blasts seen in TAM, ${ }^{20}{ }^{21}$ and in the FrenchAmerican-British (FAB) classification they are typed as AML M7 (acute megakaryoblastic leukaemia). Occasionally, other FAB types (M0, Ml and M2) are identified. ${ }^{22}$

\section{Treatment}

For many years, children with Down syndrome received suboptimal treatment and had poor survival. ${ }^{17}$ However, following recognition of the favourable response to chemotherapy, there has been increasing recruitment to national leukaemia chemotherapy protocols. The basis of the favourable response is primarily increased sensitivity of the ML-DS blasts to cytarabine (reviewed by Taub and $\mathrm{Ge}^{24}$ ). Contemporary regimens produce 5 -year survival rates of $\sim 80 \% .{ }^{17} 22{ }^{25}$ The main reason for treatment failure is toxicity (resistant disease and relapse are rare), predominantly due to mucositis and infection. Thus, current studies aim to reduce treatment intensity when compared with children with acute myeloid leukaemia who do not have Down syndrome.

\section{LINK BETWEEN TAM AND ML-DS}

There is clear evidence that TAM and ML-DS are linked conditions, with TAM the preleukaemic phase of ML-DS. TAM and ML-DS blast cells have near identical morphology, immunophenotype and ultrastructure. More definitively, in virtually all cases of TAM and ML-DS, but not other Down syndrome and non-Down syndrome leukaemias, acquired somatic mutations are present in one copy of the key megakaryocyte-erythroid transcription factor GATAl. ${ }^{911-13}{ }^{26}$ In cases of TAM that progress to ML-DS, the same GATAl mutations have been found in both TAM and ML-DS, demonstrating the two disorders have a common cell of origin. ${ }^{13}$ This strongly supports the concept that TAM and ML-DS is a unique and highly informative model of preleukaemia and leukaemia in which some key pathogenetic events are known.

TAM and ML-DS: a multi-step model of leukaemogenesis Our current understanding suggests that there are at least three distinct pathogenetic steps in ML-DS (reviewed by Vyas and Roberts $^{27}$ ).

(1) First, a fetal haemopoietic cell has to be trisomic for chromosome 21. The importance of trisomy is underscored by rare cases of TAM in mosaic DS neonates. ${ }^{28}$ Although presumably increased expression of one or more genes on 
chromosome $2 \mathrm{l}$ is important, the exact mechanism why trisomy is critical is unclear.

(2) The second required event is acquisition of a GATAl mutation that results in production of a $\mathrm{N}$-terminal truncated GATAl protein. As GATAl is encoded on the Xchromosome, in both males and females (due to $\mathrm{X}$ inactivation) only the mutant $\mathrm{N}$-terminal GATAl is expressed. It is likely that GATAls is a weak oncogene ${ }^{29-32}$ that fails to control excessive megakaryocyte differentiation.

(3) As not all cases of TAM progress to ML-DS, additional, as yet unidentified, genetic or epigenetic events are required for progression to ML-DS. Presumably, in cases where these mutations are not acquired the TAM clone extinguishes.

An outstanding issue is why patients who progress from TAM to ML-DS often develop an intervening MDS phase. Although there is a paucity of data on this, as children with Down syndrome and the MDS phase progress to acute myeloid leukaemia, ${ }^{33}$ it is likely that the MDS phase may be the clinical manifestation of progressive acquisition of additional (epi)genetic mutations.

\section{ACUTE LYMPHOBLASTIC LEUKAEMIA OF DOWN SYNDROME (ALL-DS) Clinical and laboratory presentation}

As acute lymphoblastic leukaemia is much more common in children than acute myeloid leukaemia, ALL-DS is 1.7 times more frequent than ML-DS. ${ }^{19}$ Data from national studies of childhood acute lymphoblastic leukaemia ${ }^{34-37}$ indicate that clinical features of acute lymphoblastic leukaemia are similar in children with or without Down syndrome. Most children $(>90 \%)$ have a precursor B-cell immunophenotype (CD79a+, CDl0+, CD19+), and $\mathrm{T}$ cell disease is uncommon. The laboratory findings generally overlap those of children without Down syndrome, but in Down syndrome, acute lymphoblastic leukaemia is more likely to have an adverse (hypodiploidy) rather than favourable prognostic karyotype (high hyperdiploidy and $\mathrm{t}(12: 21))$. Half the children have a normal karyotype and reciprocal translocations are rare.

\section{Treatment}

Similar to treatment for ML-DS, the prognosis of children with Down syndrome and acute lymphoblastic leukaemia improved once clinicians became convinced of the appropriateness of optimal treatment. After this, children with Down syndrome benefited from the overall improvements observed for all childhood acute lymphoblastic leukaemia. However, although most children with ALL-DS are cured $(60-70 \%)$ they have a poorer outcome than children without Down syndrome with acute lymphoblastic leukaemia (75-85\%). In recent years, the intensity of acute lymphoblastic leukaemia treatment has been escalated, and although this has benefited children without Down syndrome who have acute lymphoblastic leukaemia, children with ALL-DS have had increased major treatmentrelated toxicity. ${ }^{22}$ In contrast with ML-DS, there is no evidence of increased sensitivity of the leukaemia to treatments, but the increased risk of treatment toxicity (especially mucositis and infection) has resulted in increased treatment-related deaths. Most deaths have occurred in anthracycline-containing phases of treatment (induction and delayed intensifications). Given the risk of relapse in leukaemia, dose reduction in children with ALL-DS to reduce treatment toxicity is unattractive. The emphasis now is on attempts to improve supportive care. Whether this will sufficiently deal with the problem is as yet not known. Progress in the future will ultimately depend on a better basic understanding of the biology of acute lymphoblastic leukaemia. In this regard much less is known about the genetic or epigenetic leukaemogenic events in this condition than for TAM and ML-DS.

\section{SUMMARY}

Children with Down syndrome are predisposed to haematological disorders, especially leukaemia. Tremendous progress over the past two decades has allowed us to appreciate the distinct clinical and biological nature of myeloid and lymphoid leukaemia in children with Down syndrome. The vast majority of children with ML-DS are cured. TAM and ML-DS are unique paired clonal myeloid preleukaemic and leukaemic disorders that provide a powerful model to understand the general principles of leukaemogenesis: both preleukaemic and leukaemic conditions are easily ascertainable; two of the key genetic events (trisomy 21 and mutation of GATAl) are known; and the tools to dissect perturbed megakaryocyte and erythroid differentiation in these disorders are available. Although less progress has been made on ALL-DS, it is highly likely that this will be rectified by the ongoing research. Finally, if we can understand the biological role of trisomy 21 in these conditions it may provide an example of how altered gene dosage in human aneuploidy causes disease.

\section{ACKNOWLEDGEMENTS}

The restrictions on article size has meant important work and references have been omitted. These omissions do not reflect on the quality and importance of the research in these areas.

\section{Authors' affiliations}

David Webb, Department of Haematology, Great Ormond Street Hospital for Children, London, England

Irene Roberts, Department of Haematology, Hammersmith and St Mary's Hospitals, Imperial College, London, England

Paresh Vyas, Department of Haematology and MRC Molecular

Haematology Unit, John Radcliffe Hospital and Weatherall Institute of Molecular Medicine, Oxford, England

PV is a Wellcome Trust Senior Clinical Fellow and is funded by the Wellcome Trust, Leukaemia Research Fund and the Medical Research Council. IR is funded by the Kay Kendall Fund.

Competing interests: None.

\section{REFERENCES}

1 Miller M, Cosgriff JM. Hematological abnormalities in newborn infants with Down syndrome. Am J Med Genet 1983;16:173-7.

2 David O, Fiorucci G, Tosi M, et al. Hematological studies in children with Down syndrome. Pediatr Hematol Oncol 1996;13:271-5.

3 de Hingh YC, van der Vossen PW, Gemen EF, et al. Intrinsic abnormalities of lymphocyte counts in children with down syndrome. J Pediatr 2005;147:744-7.

4 Watts TL, Murray NA, Roberts IA. Thrombopoietin has a primary role in the regulation of platelet production in preterm babies. Pediatr Res 1999;46:28-32.

5 Richards M, Welch J, Watmore A, et al. Trisomy 21 associated transient neonatal myeloproliferation in the absence of Down's syndrome. Arch Dis Child Fetal Neonatal Ed 1998;79:F215-7.

6 Choong K, Freedman MH, Chitayat D, et al. Juvenile myelomonocytic leukemia and Noonan syndrome. J Pediatr Hematol Oncol 1999:21:523-7.

7 Zipursky A. Transient leukaemia-a benign form of leukaemia in newborn infants with trisomy 21. Br J Haematol 2003;120:930-8.

8 Massey GV, Zipursky A, Chang MN, et al. A prospective study of the natural history of transient leukemia (TL) in neonates with Down syndrome (DS): Children's Oncology Group (COG) study POG-9481. Blood 2006;107:4606-13

9 Wechsler J, Greene M, McDevitt MA, et al. Acquired mutations in GATA1 in the megakaryoblastic leukemia of Down syndrome. Nat Genet 2002;32:148-52.

10 Hitzler JK, Cheung J, Li Y, et al. GATA1 mutations in transient leukemia and acute megakaryoblastic leukemia of Down syndrome. Blood 2003;101:4301-4.

11 Groet J, McElwaine S, Spinelli M, et al. Acquired mutations in GATA1 in neonates with Down's syndrome with transient myeloid disorder. Lancet 2003;361:1617-20.

12 Rainis L, Bercovich D, Strehl S, et al. Mutations in exon 2 of GATA1 are early events in megakaryocytic malignancies associated with trisomy 21 . Blood 2003; 102:981-6.

13 Ahmed M, Sternberg A, Hall G, et al. Natural history of GATAl mutations in Down syndrome. Blood 2004;103:2480-9. 
14 Robertson M, De Jong G, Mansvelt E. Prenatal diagnosis of congenital leukemia in a fetus at 25 weeks' gestation with Down syndrome: case report and review of the literature. Ultrasound Obstet Gynecol 2003;21:486-9.

15 Kivivuori SM, Rajantie J, Siimes MA. Peripheral blood cell counts in infants with Down's syndrome. Clin Genet 1996:49:15-9.

16 Hasle $\mathrm{H}$, Niemeyer CM, Chessells JM, et al. A pediatric approach to the WHO classification of myelodysplastic and myeloproliferative diseases. Leukemia 2003;17:277-82.

17 Creutzig U, Reinhardt D, Diekamp S, et al. AML patients with Down syndrome have a high cure rate with AML-BFM therapy with reduced dose intensity. Leukemia 2005; 19:1355-60.

18 Zipursky A, Poon A, Doyle J. Leukemia in Down syndrome: a review. Pediatr Hematol Oncol 1992;9:139-49.

19 Hasle H, Clemmensen IH, Mikkelsen M. Risks of leukaemia and solid tumours in individuals with Down's syndrome. Lancet 2000;355:165-9.

20 Creutzig U, Ritter J, Vormoor J, et al. Myelodysplasia and acute myelogenous leukemia in Down's syndrome. A report of 40 children of the AML-BFM Study Group. Leukemia 1996;10:1677-86.

21 Lange BJ, Kobrinsky N, Barnard DR, et al. Distinctive demography, biology, and outcome of acute myeloid leukemia and myelodysplastic syndrome in children with Down syndrome: Children's Cancer Group Studies 2861 and 2891. Blood 1998;91:608-15.

22 Zeller B, Gustafsson G, Forestier E, et al. Acute leukaemia in children with Down syndrome: a population-based Nordic study. Br J Haematol 2005;1 28:797-804.

23 Craze JL, Harrison G, Wheatley K, et al. Improved outcome of acute myeloid leukaemia in Down's syndrome. Arch Dis Child 1999:81:32-7.

24 Taub JW, Ge Y. Down syndrome, drug metabolism and chromosome 21. Pediatr Blood Cancer 2005;44:33-9.

25 Rao A, Hills RK, Stiller C, et al. Treatment for myeloid leukaemia of Down syndrome: population-based experience in the UK and results from the Medical Research Council AML 10 and AML 12 trials. Br J Haematol 2006;132:576-83.

26 Xu G, Nagano M, Kanezaki R, et al. Frequent mutations in the GATA-1 gene in the transient myeloproliferative disorder of Down syndrome. Blood 2003; 102:2960-8
27 Vyas P, Roberts I. Down myeloid disorders: a paradigm for childhood preleukaemia and leukaemia and insights into normal megakaryopoiesis. Early Hum Dev 2006;82:767-73.

28 Carpenter E, Valverde-Garduno V, Sternberg A, et al. GATA1 mutation and trisomy 21 are required only in haematopoietic cells for development of transient myeloproliferative disorder. Br J Haematol 2005;128:548-51.

29 Kuhl C, Atzberger A, Iborra F, B. N, C. P, P. V. GATA1-mediated megakaryocyte differentiation and growth control can be uncoupled and mapped to different domains in GATA1. Mol Cell Biol 2005;25:8592-606.

30 Li Z, Godinho FJ, Klusmann JH, et al. Developmental stage-selective effect of somatically mutated leukemogenic transcription factor GATA1. Nat Genet 2005;37:613-9.

31 Muntean AG, Crispino JD. Differential requirements for the activation domain and FOG-interaction surface of GATA- 1 in megakaryocyte gene expression and development. Blood 2005; 106:1223-31.

32 Stachura DL, Chou ST, Weiss MJ. Early block to erythromegakaryocytic development conferred by loss of transcription factor GATA-1. Blood 2006; 107:87-97

33 Webb DK. Optimizing therapy for myeloid disorders of Down syndrome. Br J Haematol 2005;131:3-7.

34 Ragab AH, Abdel-Mageed A, Shuster JJ, et al. Clinical characteristics and treatment outcome of children with acute lymphocytic leukemia and Down's syndrome. A Pediatric Oncology Group study. Cancer 1991;67:1057-63.

35 Dordelmann M, Schrappe M, Reiter A, et al. Down's syndrome in childhood acute lymphoblastic leukemia: clinical characteristics and treatment outcome in four consecutive BFM trials. Berlin-Frankfurt-Munster Group. Leukemia $1998 ; 12: 645-51$

36 Chessells JM, Harrison G, Richards SM, et al. Down's syndrome and acute lymphoblastic leukaemia: clinical features and response to treatment. Arch Dis Child 2001;85:321-5

37 Whitlock JA, Sather HN, Gaynon P, et al. Clinical characteristics and outcome of children with Down syndrome and acute lymphoblastic leukemia: a Children's Cancer Group study. Blood 2005; 106:4043-9.

\section{bmjupdates+}

bmjupdates+ is a unique and free alerting service, designed to keep you up to date with the medical literature that is truly important to your practice.

bmjupdates+ will alert you to important new research and will provide you with the best new evidence concerning important advances in health care, tailored to your medical interests and time demands.

Where does the information come from?

bmjupdates+ applies an expert critical appraisal filter to over 100 top medical journals A panel of over 2000 physicians find the few 'must read' studies for each area of clinical interest

Sign up to receive your tailored email alerts, searching access and more...

www.bmjupdates.com 